Article

\title{
A Numerical Study of the Fractional Order Dynamical Nonlinear Susceptible Infected and Quarantine Differential Model Using the Stochastic Numerical Approach
}

\author{
Thongchai Botmart ${ }^{1}$ (D), Zulqurnain Sabir ${ }^{2}$, Muhammad Asif Zahoor Raja ${ }^{3}$, Wajaree Weera ${ }^{1}$, Rahma Sadat ${ }^{4}$ \\ and Mohamed R. Ali $5,6, * \mathbb{D}$ \\ 1 Department of Mathematics, Faculty of Science, Khon Kaen University, Khon Kaen 40002, Thailand; \\ thongbo@kku.ac.th (T.B.); wajawe@kku.ac.th (W.W.) \\ 2 Department of Mathematics and Statistics, Hazara University, Mansehra 21300, Pakistan; \\ zulqurnain_maths@hu.edu.pk \\ 3 Future Technology Research Center, National Yunlin University of Science and Technology, \\ 123 University Road, Section 3, Douliou, Yunlin 64002, Taiwan; rajamaz@yuntect.edu.tw \\ 4 Department of Mathematics, Zagazig Faculty of Engineering, Zagazig University, Ismailia 44519, Egypt; \\ r.mosa@zu.edu.eg \\ 5 Faculty of Engineering and Technology, Future University, Cairo 11835, Egypt \\ 6 Department of Basic Science, Faculty of Engineering at Benha, Benha University, Benha 13512, Egypt \\ * Correspondence: mohamed.reda@fue.edu.eg or mohamed.reda@bhit.bu.edu.eg
}

Citation: Botmart, T.; Sabir, Z.; Raja, M.A.Z.; Weera, W.; Sadat, R.; Ali,

M.R. A Numerical Study of the Fractional Order Dynamical Nonlinear Susceptible Infected and Quarantine Differential Model Using the Stochastic Numerical Approach. Fractal Fract. 2022, 6, 139. https:// doi.org/10.3390/fractalfract6030139 Academic Editors: António M. Lopes, Alireza Alfi, Liping Chen and Sergio A. David

Received: 20 December 2021 Accepted: 24 February 2022 Published: 1 March 2022

Publisher's Note: MDPI stays neutral with regard to jurisdictional claims in published maps and institutional affiliations.

Copyright: (C) 2022 by the authors. Licensee MDPI, Basel, Switzerland. This article is an open access article distributed under the terms and conditions of the Creative Commons Attribution (CC BY) license (https:// creativecommons.org/licenses/by/ $4.0 /)$.

\begin{abstract}
The theme of this study is to present the impacts and importance of the fractional order derivatives of the susceptible, infected and quarantine (SIQ) model based on the coronavirus with the lockdown effects. The purpose of these investigations is to achieve more accuracy with the use of fractional derivatives in the SIQ model. The integer, nonlinear mathematical SIQ system with the lockdown effects is also provided in this study. The lockdown effects are categorized into the dynamics of the susceptible, infective and quarantine, generally known as SIQ mathematical system. The fractional order SIQ mathematical system has never been presented before, nor solved by using the strength of the stochastic solvers. The stochastic solvers based on the Levenberg-Marquardt backpropagation scheme (LMBS) along with the neural networks (NNs), i.e., LMBS-NNs have been implemented to solve the fractional order SIQ mathematical system. Three cases using different values of the fractional order have been provided to solve the fractional order SIQ mathematical model. The data to present the numerical solutions of the fractional order SIQ mathematical model is selected as $80 \%$ for training and $10 \%$ for both testing and validation. For the correctness of the LMBS-NNs, the obtained numerical results have been compared with the reference solutions through the Adams-Bashforth-Moulton based numerical solver. In order to authenticate the competence, consistency, validity, capability and exactness of the LMB-NNs, the numerical performances using the state transitions (STs), regression, correlation, mean square error (MSE) and error histograms (EHs) are also provided.
\end{abstract}

Keywords: SIQ mathematical model; fractional order; coronavirus; Levenberg-Marquardt backpropagation scheme; neural networks; Adams-Bashforth-Moulton

\section{Introduction}

There are a number of dangerous and transmitted diseases like dengue, HIV and Ebola [1-3]. The coronavirus is a transmitted disease and has played a significant role in human lives for the last two years. It badly affected the economies, industries, sports, social activities, education sectors and each part of life [4,5]. The coronavirus disease spread quickly, and a number of casualties happened in a short time. The basic role of the coronavirus spreading is due to travel or transportations of individuals from defective countries to different areas [6,7]. The vaccination process was started as a hope to control 
this series viral disease. It is stated in the literature that the individual's migration has a vital role in the spreading of the infection. It has also been noted that immigration is not only an issue of the infections, but the other reasons may also affect the spread of the virus [8].

A number of the approaches have been used to solve the mathematical form of the coronavirus along with different features. Rhodes et al. [9] proposed the mathematical ODEs for the communal distresses due to coronavirus. Benvenuto et al. [10] implemented the ARIMA system for the coronavirus. Mustafa et al. [11] presented a mathematical system to forecast and analyze the coronavirus transmission. Sivakumar [12] analyzed the predictive control for the coronavirus in India. Nesteruk [13] assessed the dynamics of the coronavirus pandemic in Ukraine using the double data sets. Thompson [14] studied the epidemiologic system with the use of significant apparatus using the coronavirus interferences. Libotte [15] presented an administration plan for the coronavirus vaccine. Sadiq et al. [16] investigated the impacts of nanomaterial to handle the coronavirus disease. Gumel et al. [17] discovered a mathematical system for the coronavirus disease. Ortenzi et al. [18] presented a transdisciplinary discipline study of coronavirus in Italy. Sánchez et al. [19] designed a susceptible, infected, treatment and recovered (SITR) mathematical model using the sense of corona virus. In other studies, Sabir et al. [20] provided the stochastic performances of the SITR model-based coronavirus. Moore et al. [21] designed a mathematical coronavirus system to investigate a vaccination impact and a non-pharmaceutical intervention. Umar [22] studied theoretical performances to treat coronavirus. Anirudh [23] provided the transmission dynamics prediction based on the coronavirus. Chen et al. [24] provided the social distance effects using the mathematical form of the dynamics of coronavirus. Zhang et al. [25] expressed the coronavirus dynamics using the stochastic perturbation behavior. Soumia et al. [26] described the possible inhibitors of coronavirus.

In this study, the fractional order derivatives of susceptible, infected and quarantine (SIQ) model based on the coronavirus with the lockdown effects are presented using the stochastic numerical performances of the Levenberg-Marquardt backpropagation scheme (LMBS) along with the neural networks (NNs), i.e., LMBS-NNs.

The design of the fractional order SIQ model is presented in Section 2. The details of the stochastic applications are provided in Section 3. The LMBS-NNs structure is explained in Section 4. The simulations of the fractional order SIQ model are provided in Section 5. Finally, the conclusion is drawn in the Section 6.

\section{Mathematical Design of the Fractional Order SIQ System}

In this section, the lockdown impacts as protective measures have been provided in the SIQ model. The lockdown effects are categorized into the dynamics of the susceptible, infective and quarantine classes-based system of differential equations. The mathematical form of the SIQ model is provided as [27]:

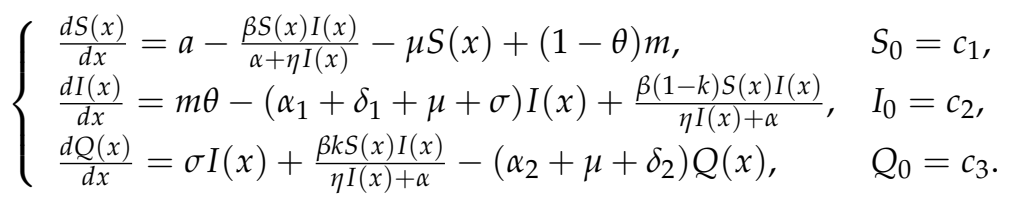

The necessary and exhaustive detail of the SIQ mathematical model together with the description of each parameter is given in Table 1. Moreover, the selection of the appropriate values given in the system (1) provided in [27] along with the theoretical details of optimal control, global and local stabilities. 
Table 1. Description of each comprehensive detail of each specification of the SIQ mathematical model.

\begin{tabular}{cc}
\hline Parameters & Details \\
\hline$a$ & Recruitment rate \\
$\alpha$ & Half saturation constant \\
$\eta$ & Positive value \\
$m$ & Migrants number \\
$\beta$ & Transmission infection rate \\
$\mu$ & Natural death rate \\
$\delta_{1}$ & Recovery of infective population \\
$k$ & Contact tracing rate \\
$\theta$ & Infected migrants' rate \\
$\sigma$ & 0.59 per day \\
$\alpha_{2}$ & Disease associated quarantine's population death rate \\
$\alpha_{1}$ & Disease related infective population's death rate \\
$\delta_{1}, c_{2}$ and $c_{3}$ & Quarantined population recovered rate \\
& Contents: Initial conditions (ICs) \\
\hline
\end{tabular}

In the current study, the numerical investigations of the fractional order derivatives of SIQ model based on the coronavirus with the lockdown effects (1) have been provided by using the artificial intelligence (AI) with the design of LMBS-NNs. The design of the fractional order derivatives of SIQ model is formulated for the in-depth analysis of the super slow evolution as well as superfast transitions by replacing the ordinary integer order derivation in set of Equation (1) by fractional orders. The modified system (1) is given as follows:

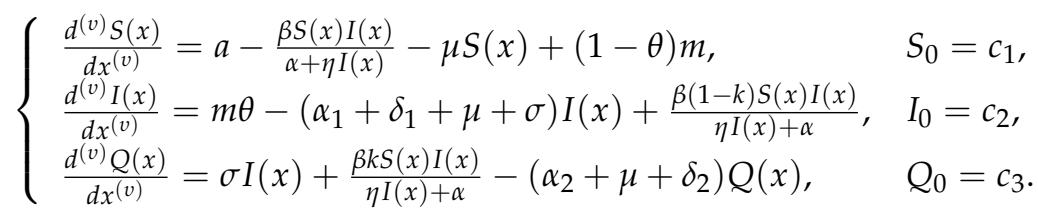

where $v$ shows the FO derivative in the above system.

\section{Novel Topographies and Outline of the Stochastic Solvers}

The numerical stochastic operators through the LMBS-NNs are provided to solve the fractional order derivatives of SIQ model based on the coronavirus with the lockdown effects. The local and global operator performances through the stochastic computing solvers have been exploited to solve the numerous nonlinear, complex, stiff and singular systems [28].

The aim of this study is to perform the numerical representations of the fractional order derivatives of SIQ model based on the coronavirus with the lockdown effects using the stochastic procedures of the LMBS-NNs. It is observed that the time-fractional order derivatives have a number of applications to define the system conditions. The derivative order form represents the remembrance, but the memory function represents the derivative of fractional order. These fractional derivatives indicate the real-world applications [29,30]. Some novel features of the LMBS-NNs for the mathematical fractional order system using the SIQ model are presented as:

- A novel design of the fractional order SIQ model based on the coronavirus with the lockdown effects is presented;

- The stochastic measures have not been applied before to solve the fractional order SIQ model based on the coronavirus with the lockdown effects;

- The numerical investigations through the stochastic paradigms are successfully presented using the fractional order SIQ mathematical model;

- $\quad$ AI with the design of LMBS-NNs is presented to solve the nonlinear fractional order SIQ mathematical model; 
- Three different fractional order variations based on the SIQ model have been numerically solved to authenticate the reliability of the proposed scheme;

- The brilliance of the stochastic computing solver based LMBS-NNs is provided using the comparison of the obtained and reference (Adams-Bashforth-Moulton) solutions;

- The accuracy of the scheme is observed through the absolute error (AE) performances that is achieved in good order to solve the fractional order SIQ mathematical model;

- The regression, STs, MSE and EHs and correlation performances approve the dependability and constancy of the designed LMBS-NNs to solve the fractional order SIQ mathematical model.

\section{Proposed Procedures: LMBS-NNs}

The proposed LMBS-NNs structure for solving the fractional order SIQ mathematical model is explained in this section. The methodology is designed in two parts. First, the essential performances of the LMB-NNs operator are provided. Next, the execution method via LMBS-NNs is implemented to solve the fractional order SIQ mathematical model. The proposed LMBS-NN are executed with analysis on the similar pattern as given in reported studies [31,32].

Figure 1 presents the multi-layer optimization procedures using the numerical stochastic LMBS-NNs, while the single layer neuron structure is plotted in Figure 2. The LMBSNNs procedures are provided using 'nftool' command in Matlab with the selection of data as $80 \%$ for training and $10 \%$ for both testing and authorization.

\section{Problem: Fractional order SIQ system}

Intelligent computational structure

The multi-layer nets structure through the designed LMBS-

NNs to solve the fractional order SIQ mathematical model based on the coronavirus

$$
\begin{cases}\frac{d^{(\nu)} S(x)}{d x^{(v)}}=a-\frac{\beta S(x) I(x)}{\alpha+\eta I(x)}-\mu S(x)+(1-\theta) m, & S_{0}=c_{1}, \\ \frac{d^{(\nu)} I(x)}{d x^{(\nu)}}=m \theta-\left(\alpha_{1}+\delta_{1}+\mu+\sigma\right) I(x)+\frac{\beta(1-k) S(x) I(x)}{\eta I(x)+\alpha}, & I_{0}=c_{2}, \\ \frac{d^{(\nu)} Q(x)}{d x^{(\nu)}}=\sigma I(x)+\frac{\beta k S(x) I(x)}{\eta I(x)+\alpha}-\left(\alpha_{2}+\mu+\delta_{2}\right) Q(x), & Q_{0}=c_{3} .\end{cases}
$$

Mathematical model

\section{Methodology: LMBS-NNs}

\section{Reference solutions}

A dataset is designed through the numerical computing solver for the fractional order SIQ dynamical system-based coronavirus

\section{Obtained Solutions}

Activate the designed LMBS-NNs using the reference data to find the approximate results of the fractional order SIQ dynamical system-based coronavirus

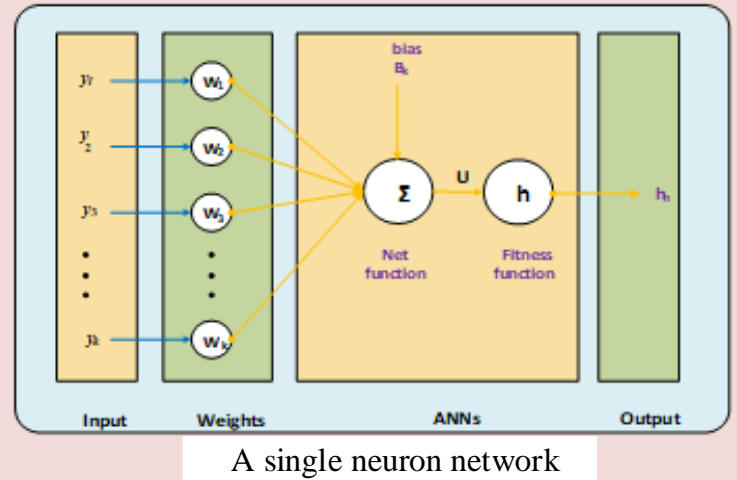

Figure 1. Cont. 


\section{Results with analysis}

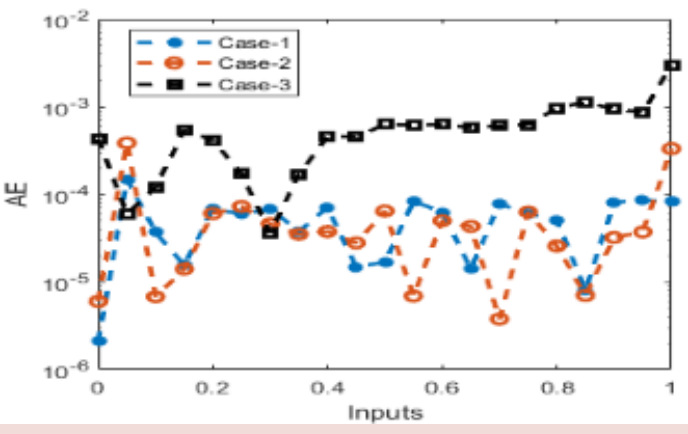

$\mathbf{A E}$

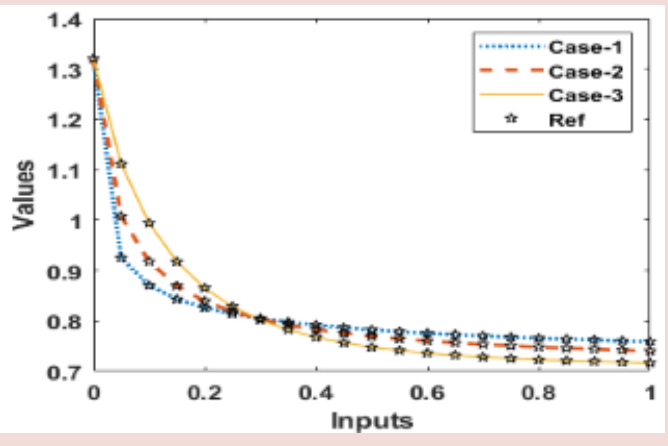

Results

Approximate LMBS-NNs solutions and investigation related to the fitness values, STs, EHs, regressions and MSE for the fractional order SIQ dynamical system-based coronavirus

Figure 1. Workflow structure of LMBS-NNs to solve the fractional order SIQ model.

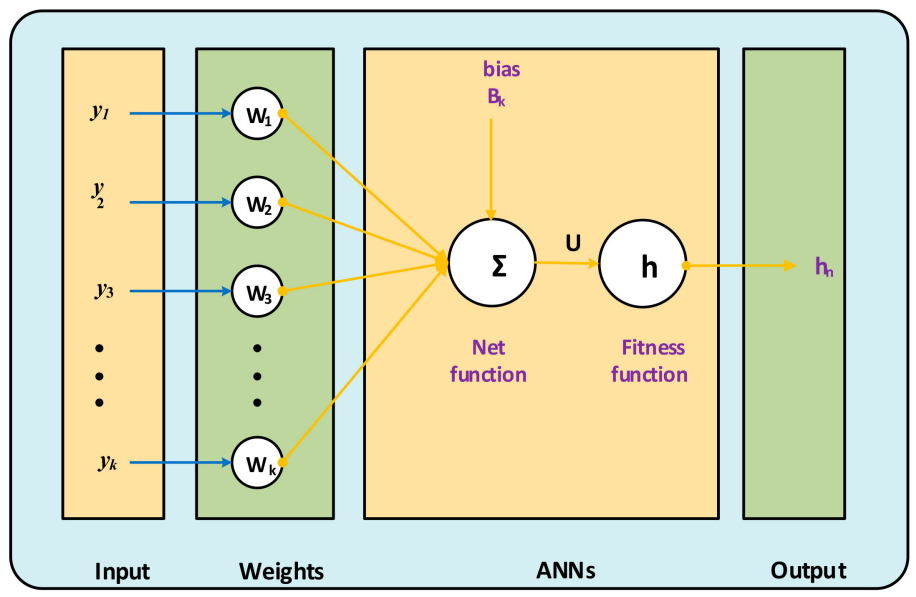

Figure 2. Construction of a single neuron.

\section{Results through the Designed Method}

This section shows the numerical performances of three different fractional order variations to solve the nonlinear SIQ mathematical system using the proposed LMBS-NNs. The mathematical representation of each variation is presented in the below cases as:

Case 1: Consider a fractional order coronavirus based SIQ mathematical model by taking the appropriate values $v=0.5, a=2.6, \beta=2.1, \alpha=5, \sigma=0.59, \eta=1, \alpha_{1}=1.78, \delta_{1}=0.4$, $\mu=5.2, \alpha_{2}=1.78, \delta_{2}=0.4, \theta=0.9, k=0.1, m=14, c_{1}=1.32, c_{2}=2.29$ and $c_{3}=3.5$ is provided as:

$$
\begin{cases}\frac{d^{(0.5)} S(x)}{d x^{(0.5)}}=4-5.2 S(x)-\frac{2.1 S(x) I(x)}{5+I(x)}, & S_{0}=1.32 \\ \frac{d^{(0.5)} I(x)}{d x^{(0.5)}}=12.6-7.97 I(x)+\frac{1.89 I(x) S(x)}{I(x)+5}, & I_{0}=2.29 \\ \frac{d^{(0.5)} Q(x)}{d x^{(0.5)}}=0.59 I(x)+\frac{0.21 S(x) I(x)}{5+I(x)}-7.38 Q(x), & Q_{0}=3.5 .\end{cases}
$$

Case 2: Consider a fractional order coronavirus based SIQ mathematical model by taking the appropriate values $v=0.7, a=2.6, \beta=2.1, \alpha=5, \sigma=0.59, \eta=1, \alpha_{1}=1.78, \delta_{1}=0.4$, 
$\mu=5.2, \alpha_{2}=1.78, \delta_{2}=0.4, \theta=0.9, k=0.1, m=14, c_{1}=1.32, c_{2}=2.29$ and $c_{3}=3.5$ is provided as:

$$
\begin{cases}\frac{d^{(0.7)} S(x)}{d x^{(0.7)}}=4-5.2 S(x)-\frac{2.1 S(x) I(x)}{5+I(x)}, & S_{0}=1.32, \\ \frac{d^{(0.7)} I(x)}{d x^{(0.7)}}=12.6-7.97 I(x)+\frac{1.89 I(x) S(x)}{I(x)+5}, & I_{0}=2.29, \\ \frac{d^{(0.7)} Q(x)}{d x^{(0.7)}}=0.59 I(x)+\frac{0.21 S(x) I(x)}{5+I(x)}-7.38 Q(x), & Q_{0}=3.5 .\end{cases}
$$

Case 3: Consider a fractional order coronavirus based SIQ mathematical model by taking the appropriate values $v=0.9, a=2.6, \beta=2.1, \alpha=5, \sigma=0.59, \eta=1, \alpha_{1}=1.78, \delta_{1}=0.4$, $\mu=5.2, \alpha_{2}=1.78, \delta_{2}=0.4, \theta=0.9, k=0.1, m=14, c_{1}=1.32, c_{2}=2.29$ and $c_{3}=3.5$ is provided as:

$$
\begin{cases}\frac{d^{(0.9)} S(x)}{d x^{(0.9)}}=4-5.2 S(x)-\frac{2.1 S(x) I(x)}{5+I(x)}, & S_{0}=1.32 \\ \frac{d^{(0.9)} I(x)}{d x^{(0.9)}}=12.6-7.97 I(x)+\frac{1.89 I(x) S(x)}{I(x)+5}, & I_{0}=2.29 \\ \frac{d^{(0.9)} Q(x)}{d x^{(0.9)}}=0.59 I(x)+\frac{0.21 S(x) I(x)}{5+I(x)}-7.38 Q(x), & Q_{0}=3.5 .\end{cases}
$$

The numerical presentations through the simulations of coronavirus based SIQ mathematical model is presented by using the stochastic LMBS-NNs procedures with 8 numbers of neurons along with the selection of data as $80 \%$ for training and $10 \%$ for both testing and authorization. The hidden, output and input neuron's structure is given in Figure 3.

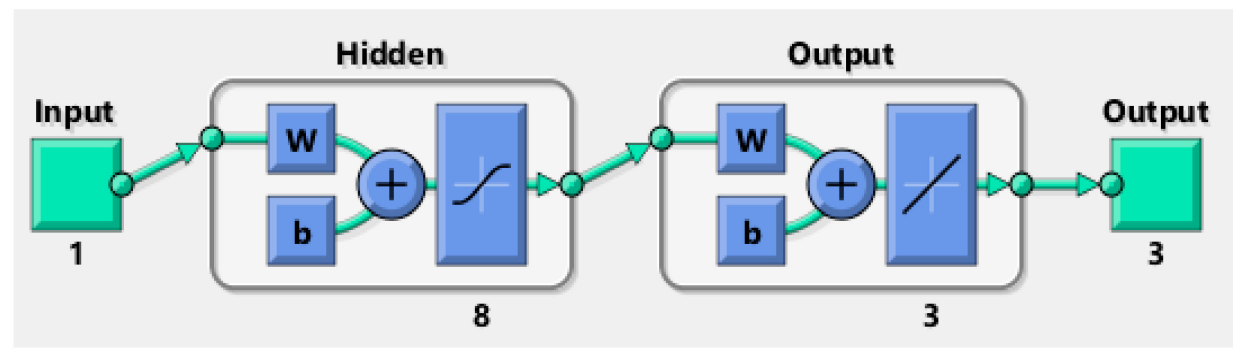

Figure 3. Proposed LMBS-NNs for the fractional order coronavirus based SIQ model.

The graphical representations are plotted in Figures 4-6 to solve the fractional order coronavirus based SIQ mathematical model by using the LMBS-NNs procedures. In order to check the best performances and STs, the graphical illustrations are provided in Figures 4 and 5. The MSE and STs values for training, best curves and authentication are derived in Figure 4 to solve the fractional order coronavirus based SIQ mathematical model. The obtained values of MSE based on the best performances of the fractional order coronavirus based SIQ mathematical model have been calculated at epochs 294, 1000 and 155 that are calculated as $1.2309 \times 10^{-8}, 5.17679 \times 10^{-9}$ and $1.9259 \times 10^{-7}$, respectively. The gradient measures are also plotted in Figure 4 to solve the fractional order coronavirus based SIQ mathematical model using the LMBS-NNs. These gradient performances have been calculated as $5.1656 \times 10^{-6}, 1.9123 \times 10^{-6}$ and $2.0104 \times 10^{-5}$ for case 1, 2 and 3. These graphical representations indicate the convergence of proposed LMBS-NNs to solve the fractional order coronavirus based SIQ mathematical model using the LMBS-NNs. Figures 5-8 represents the values of the fitting curves to solve each case of fractional order coronavirus based SIQ mathematical model. These plots represent the comparative performances of the reference and obtained results. The error plots from the substantiation, testing and training to solve each case of fractional order coronavirus based SIQ mathematical model are provided in Figure 5 (a to c) while, the EHs are plotted in Figure 5d-f. The EHs are calculated as $2.38 \times 10^{-4}, 7.10 \times 10^{-5}$ and $4.29 \times 10^{-4}$ for case 1, 2 and 3, respectively. The regression measures are provided in Figure $6 \mathrm{a}-\mathrm{c}$ based on the fractional order coronavirus based SIQ mathematical model. The correlation is 
provided to validate the regression performance in Figure 6. It is clear in understanding that the correlation plots are calculated as 1 for the fractional order coronavirus based SIQ mathematical model. The training, testing and authentication representations denote the correctness of the stochastic LMBS-NNs procedure to solve the fractional order coronavirus based SIQ mathematical model. The convergence through MSE using the complexity, training, authentication, iterations, testing and backpropagation is provided in Table 2 Figure to solve the fractional order coronavirus based SIQ mathematical model. The brief description of the parameters tabulated is provided as follows; the validation performance means that the value of fitness, i.e., MSE, for the data samples used for the validation, i.e., $10 \%$ of total samples, validation checks being the controlling paper for the networks to stop further learning of the weights, $\mathrm{Mu}$ being the adaptive Levenberg-Marquardt parameter for convergence controlling coefficient of the algorithm, gradient being the first order optimality parameter, performance means fitness on MSE and time in seconds being the time complexity of adaption of the networks.

(a)

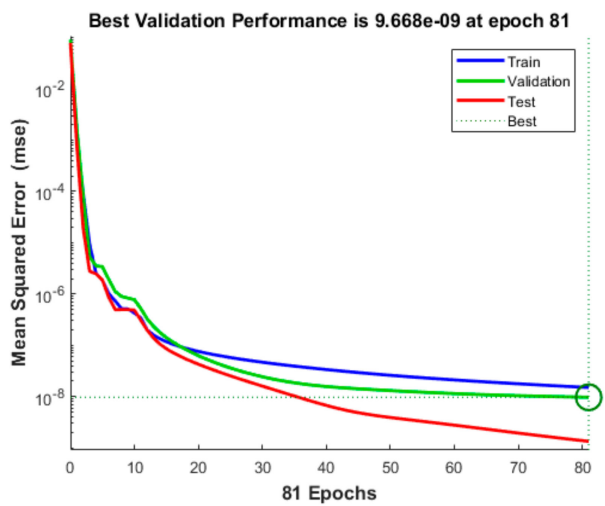

(b)

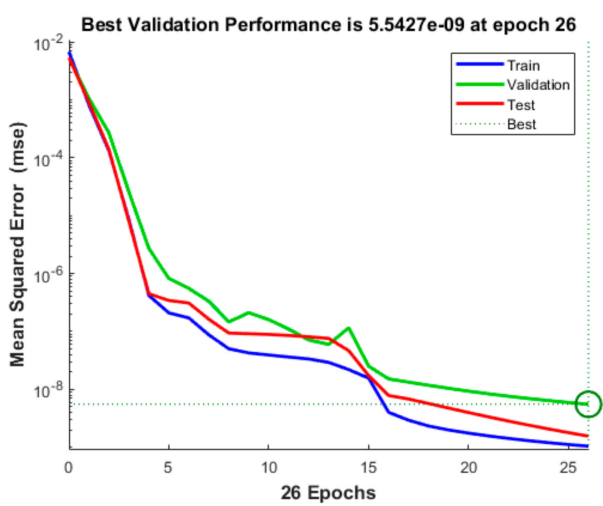

(c)

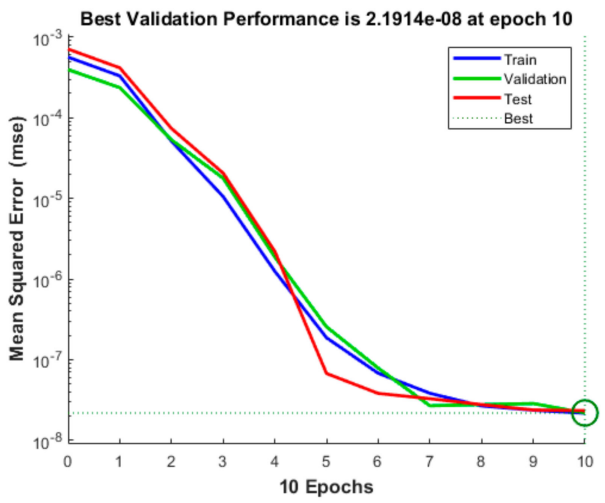

(d)
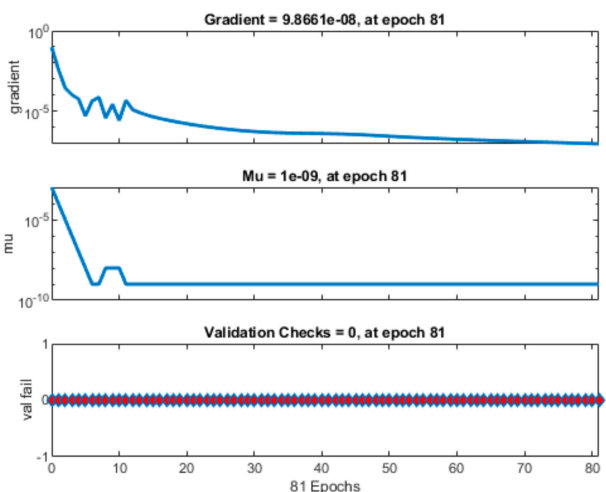

(e)
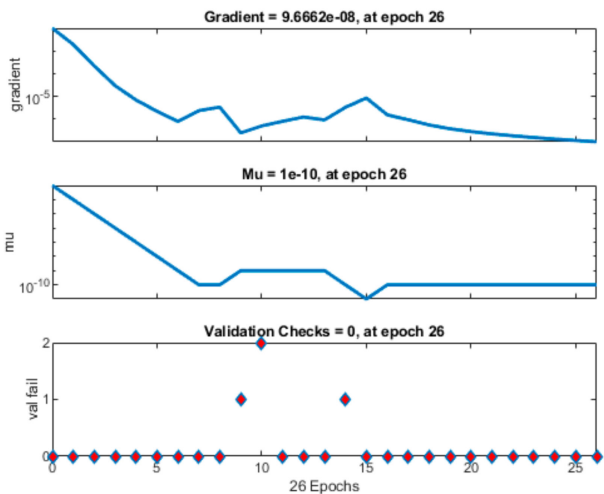

(f)

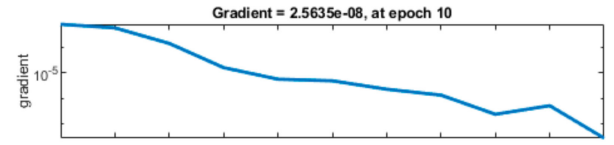

$\mathrm{Mu}=1 \mathrm{e}-09$, at epoch

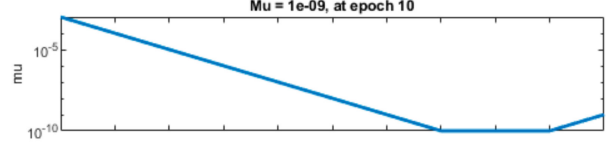

Validation Checks $=0$, at epoch

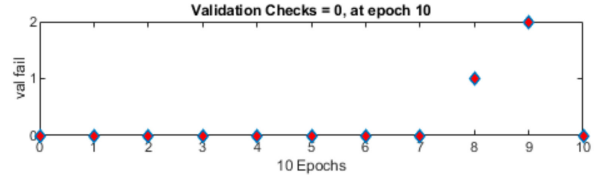

Figure 4. STs and MSE performances to solve the fractional order coronavirus based SIQ mathematical model. (a) Case 1 analysis on MSE. (b) Case 2: analysis on MSE. (c) Case 3: analysis on MSE. (d) Case I: algorithm parameter. (e) Case 2: algorithm parameter. (f) Case 3: algorithm parameter. 
(a)

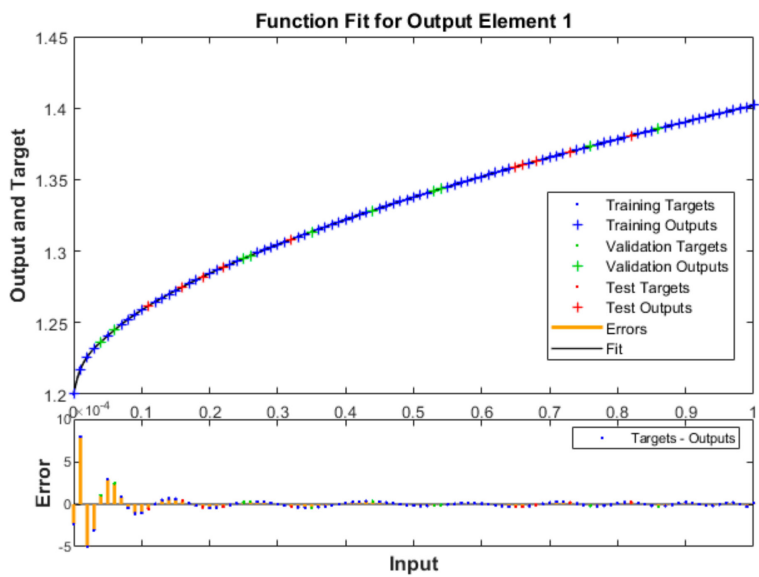

(b)

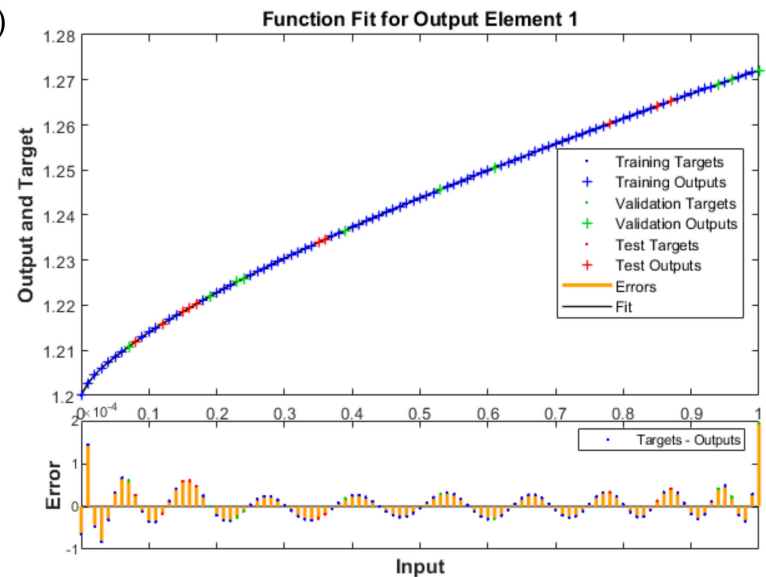

(c)

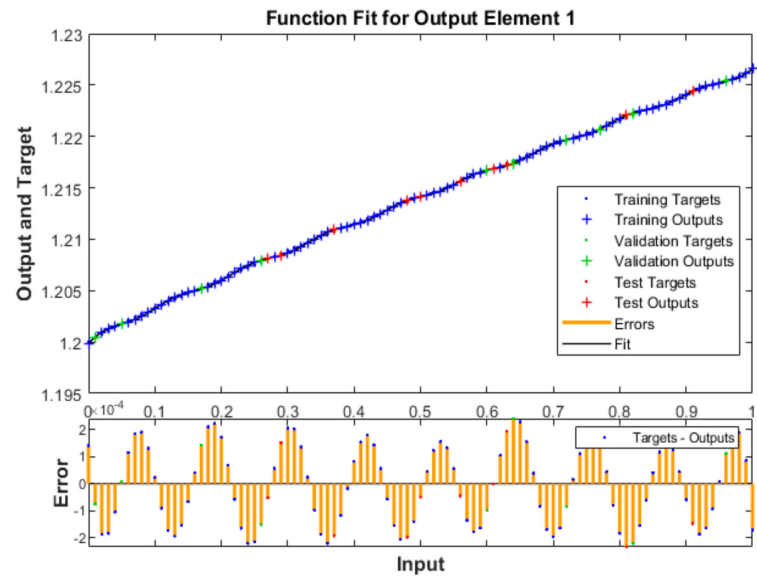

(d)

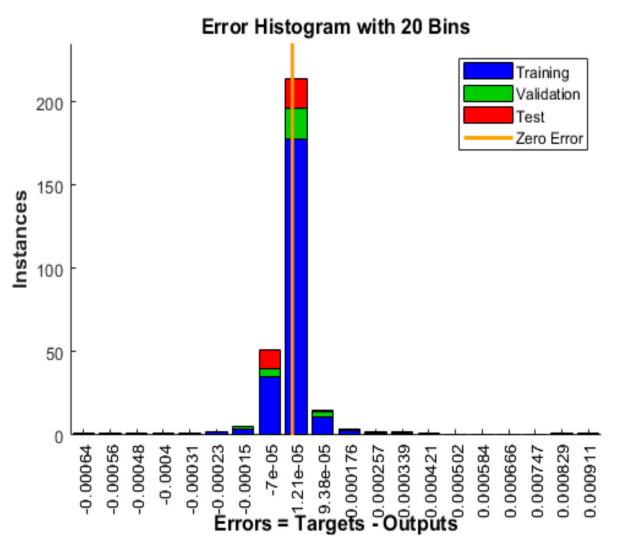

(e)

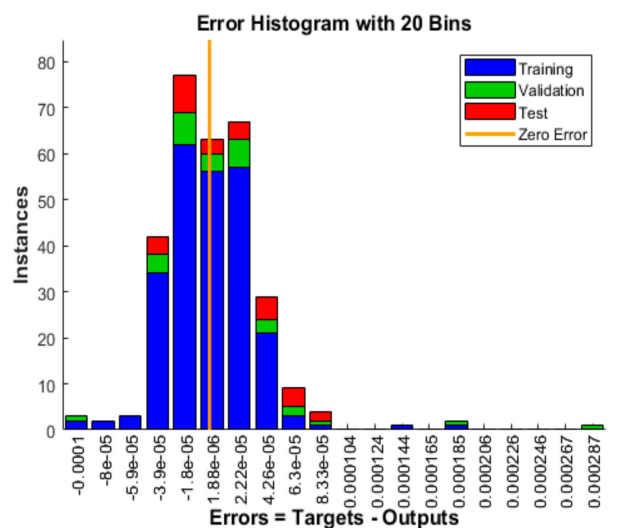

$(\mathbf{f})$

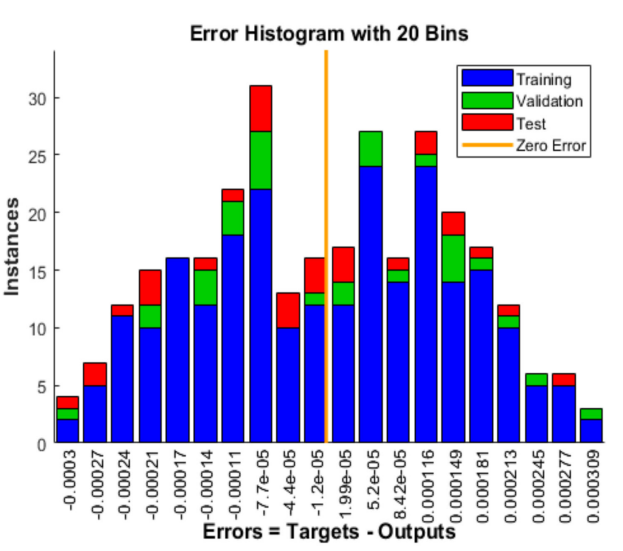

Figure 5. Valuations of the results and EHs for the STs to solve the fractional order coronavirus based SIQ mathematical model. (a) Case 1: Result assessments. (b) Case 2: Result assessments. (c) Case 3: Result assessments. (d) Case I: EH. (e) Case 2: EHs. (f) Case 3: EHs.

Table 2. LMBS-NNs procedure to solve the fractional order coronavirus based SIQ mathematical model.

\begin{tabular}{|c|c|c|c|c|c|c|c|c|}
\hline \multirow{2}{*}{ Case } & \multicolumn{3}{|c|}{ MSE } & \multirow{2}{*}{ Gradient } & \multirow{2}{*}{ Performance } & \multirow{2}{*}{ Epoch } & \multirow{2}{*}{$\mathbf{M u}$} & \multirow{2}{*}{ Time } \\
\hline & Training & Testing & Validation & & & & & \\
\hline 1 & $2.01 \times 10^{-8}$ & $4.14 \times 10^{-6}$ & $1.23 \times 10^{-8}$ & $5.17 \times 10^{-6}$ & $1.98 \times 10^{-8}$ & 300 & $1 \times 10^{-8}$ & 06 \\
\hline 2 & $2.37 \times 10^{-9}$ & $1.64 \times 10^{-7}$ & $5.17 \times 10^{-9}$ & $1.91 \times 10^{-6}$ & $2.38 \times 10^{-9}$ & 1000 & $1 \times 10^{-8}$ & 06 \\
\hline 3 & $1.45 \times 10^{-7}$ & $5.21 \times 10^{-6}$ & $1.92 \times 10^{-7}$ & $2.01 \times 10^{-5}$ & $1.37 \times 10^{-7}$ & 161 & $1 \times 10^{-7}$ & 03 \\
\hline
\end{tabular}



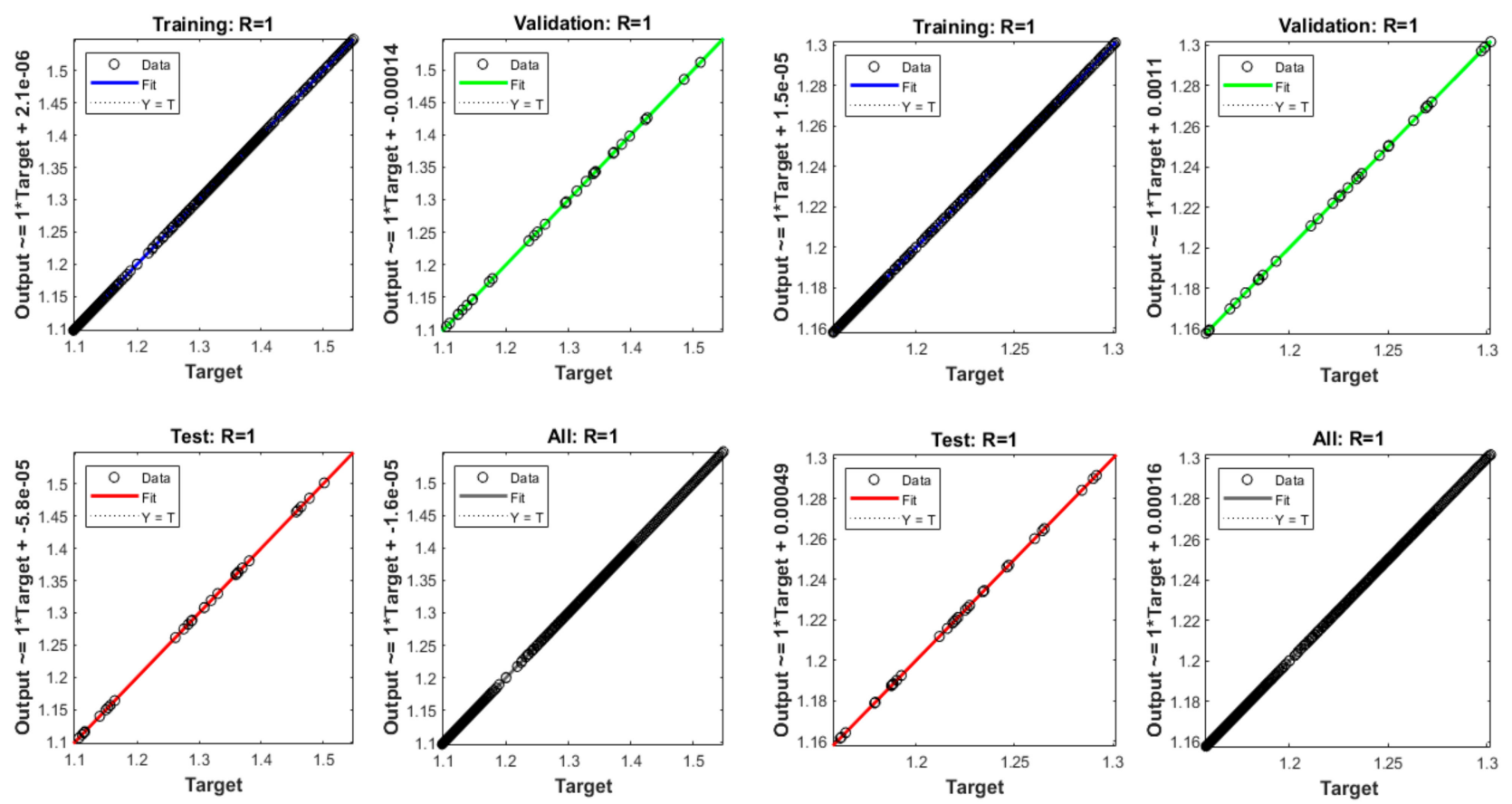

(a)
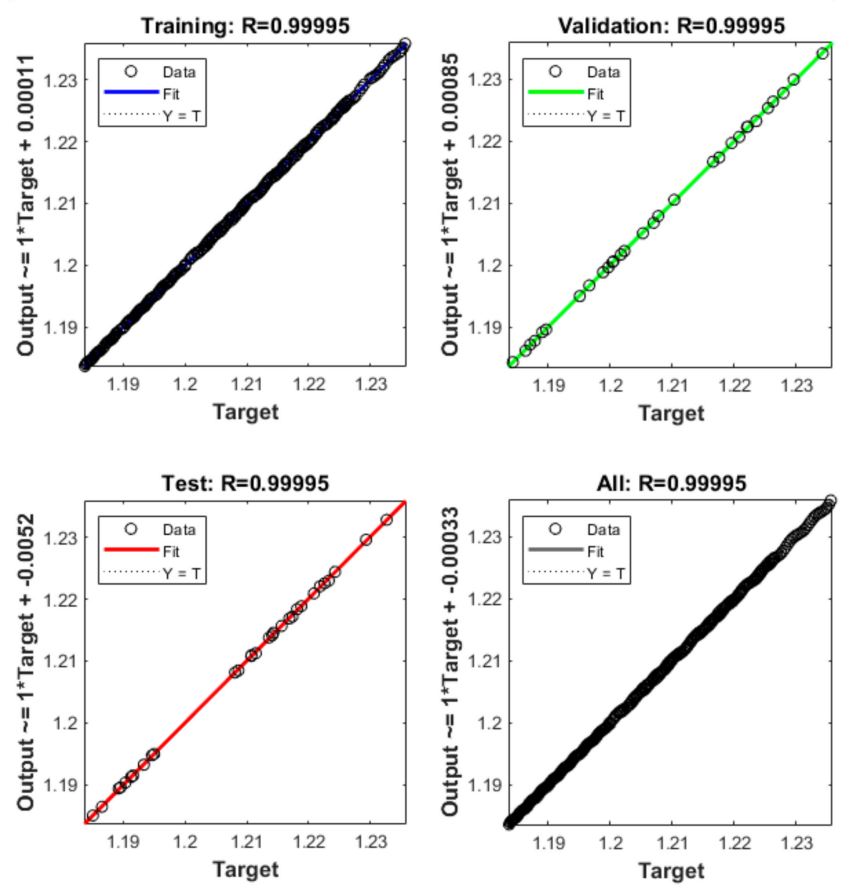

(c)

Figure 6. Regression plots STs to solve the fractional order coronavirus based SIQ mathematical model. (a) Regression plots: Case 1. (b) Regression plots: Case 2. (c) Regression plots: Case 3. 
The plots of the result comparisons and AE values are provided in Figures 7 and 8. The numerical representations are provided to solve the fractional order coronavirus based SIQ mathematical model using the stochastic LMBS-NNs. The reference and obtained numerical performances are plotted in Figure 7 through the overlapping of the results. The result overlapping authenticates the exactness of the LMBS-NNs to solve the fractional order coronavirus based SIQ mathematical model. The AE values to solve the SIQ model are performed in Figure 8. The AE for the susceptible individuals $S(x)$ calculated as $10^{-4}$ to $10^{-7}, 10^{-4}$ to $10^{-6}$ and $10^{-4}$ to $10^{-5}$ for case 1 to 3 . The AE for the infected individuals $I(x)$ calculated as $10^{-4}$ to $10^{-6}, 10^{-4}$ to $10^{-7}$ and $10^{-4}$ to $10^{-6}$ for case 1 to 3 . Similarly, the AE for the quarantine individuals $Q(x)$ calculated as $10^{-4}$ to $10^{-6}, 10^{-4}$ to $10^{-5}$ and $10^{-3}$ to $10^{-5}$ for case 1 to 3 . These AE values represent the exactness of the proposed LMBS-NNs to solve the fractional order coronavirus based SIQ mathematical model.

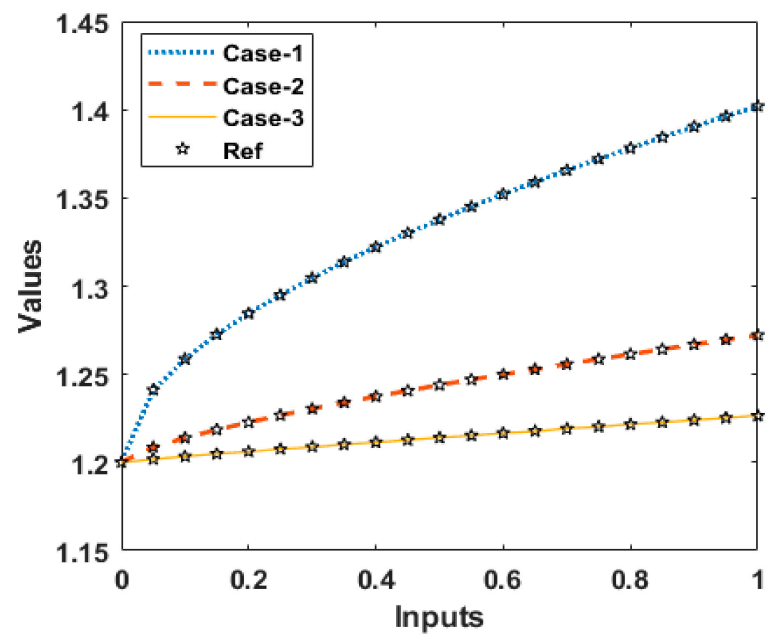

(a)

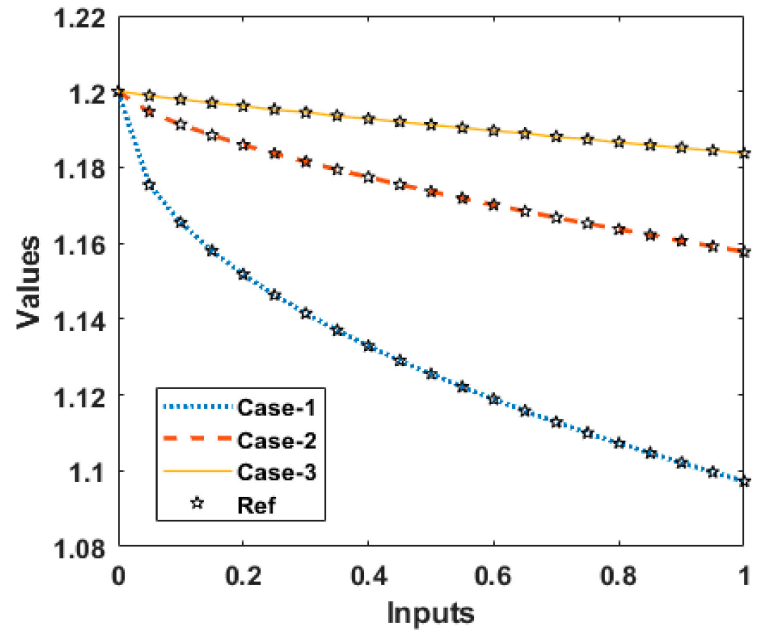

(b)

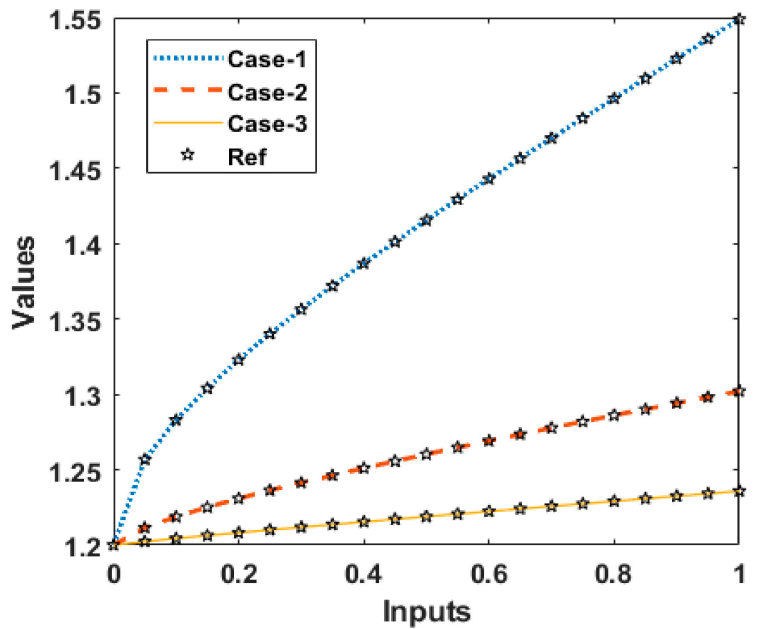

(c)

Figure 7. Results based on the fractional order coronavirus based SIQ mathematical model. (a) Results for $S(x)$. (b) Results for $I(x)$. (c) Results for $Q(x)$. 


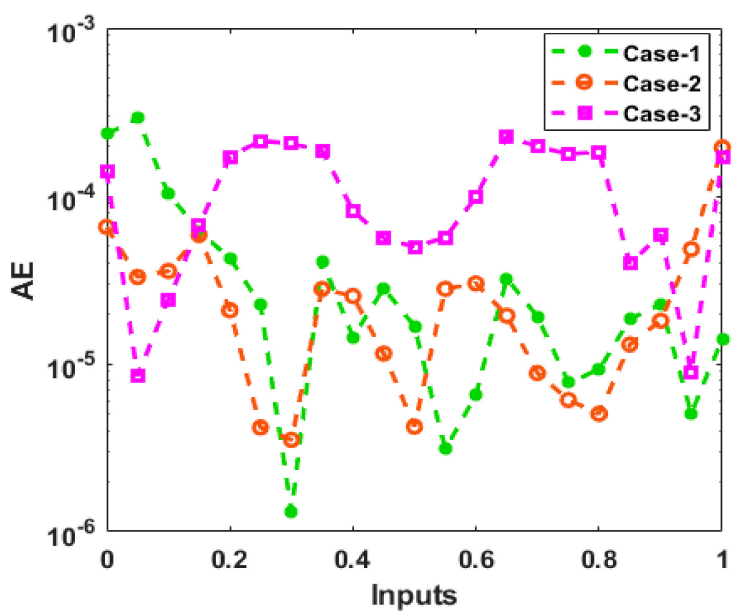

(a)

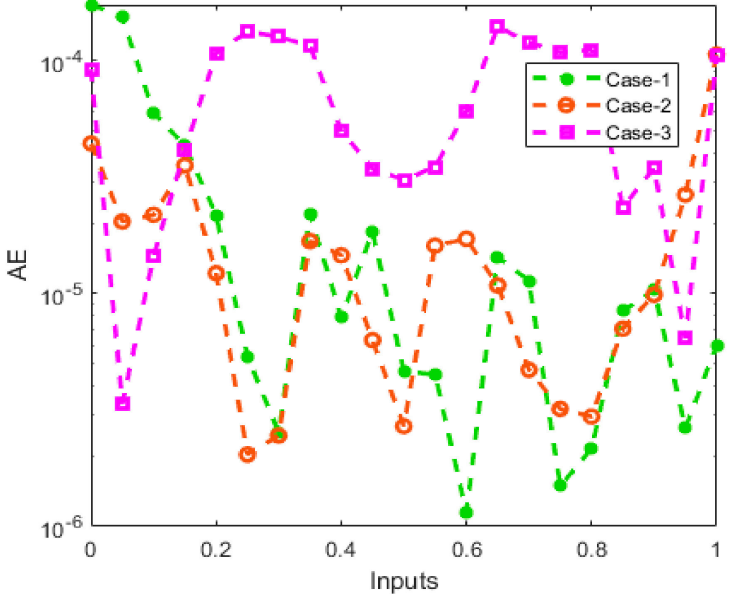

(b)

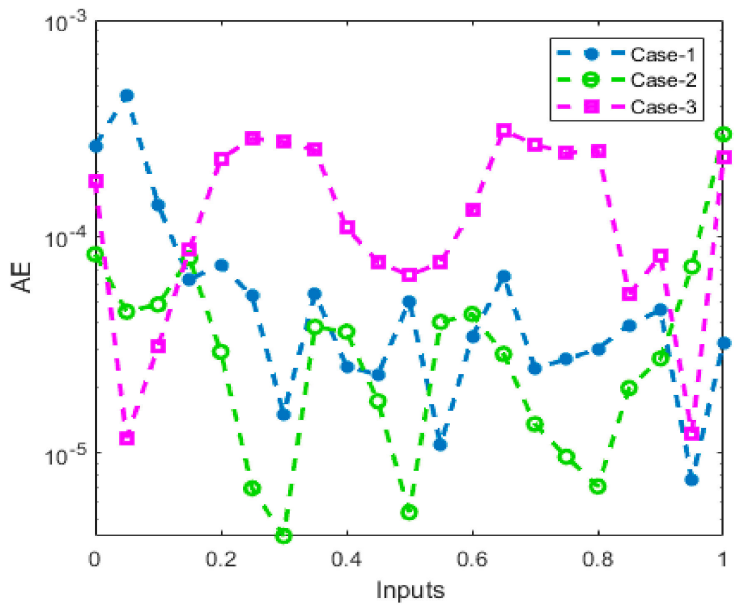

(c)

Figure 8. AE based on the fractional order coronavirus based SIQ mathematical model. (a) AE for $S(x)$. (b) AE for $I(x)$. (c) AE for $Q(x)$.

\section{Concluding Remarks}

In this work, the numerical presentations of the coronavirus based SIQ mathematical model are presented. The aim of this study is to provide the fractional order study using the dynamics of coronavirus based SIQ mathematical model to get more accurate performances of the system. The integer nonlinear mathematical SIQ system with the lockdown effects was also provided in this study. The fractional order coronavirus based SIQ mathematical model is classified into three dynamics, susceptible, infective and quarantine, generally known as the SIQ mathematical system. The numerical performances of the fractional order coronavirus based SIQ mathematical model have never been presented nor solved by using the stochastic Levenberg-Marquardt backpropagation neural networks. Three cases using different values of the fractional order have been provided to solve the fractional order SIQ mathematical model. The data to present the numerical solutions of the fractional order SIQ mathematical model were selected as $80 \%$ for training and $10 \%$ for both testing and authorization. Eight numbers of neurons were used to present the numerical performances of the fractional order SIQ mathematical system. The numerical results of the fractional order SIQ mathematical system have been compared with the Adams-Bashforth-Moulton solver. To reduce the MSE, the obtained numerical results have been performed by using the LMBS-NNs. The reliability and competence of LMBS-NNs and the numerical performances have been illustrated using the STs, regression, correlation, EHs and MSE. The correctness of the LMBS-NNs based on the fractional order SIQ mathematical model is observed via 
the matching of reference and obtained results. The performance of the scheme is verified based on the consistency and dependability of the proposed LMBS-NNs.

In future work, the LMBS-NNs can be implemented to present the numerical measures of the lonngren-wave, fluid mechanics systems, bioinformatics studies as well as information security models.

Author Contributions: T.B.: Simulate our proposed structures. Z.S.: Simulate our proposed structures. W.W.: Simulate our proposed structures. M.A.Z.R.: Do the calculations of CsPbBr3. R.S.: Do the calculations of CsPbBr3. M.R.A.: Provide the idea of this manuscript. Z.S.: Provide the idea of this manuscript. All authors have read and agreed to the published version of the manuscript.

Funding: This research received funding support from the NSRF via the Program Management Unit for Human Resources \& Institutional Development, Research and Innovation (grant number B05F640088).

Institutional Review Board Statement: Not applicable.

Informed Consent Statement: Not applicable.

Data Availability Statement: No data were used to support this study.

Conflicts of Interest: The authors declare that they have no known competing financial interest or personal relationships that could have appeared to influence the work reported in this paper.

\section{References}

1. Guerrero-Sánchez, Y.; Umar, M.; Sabir, Z.; Guirao, J.L.G.; Raja, M.A.Z. Solving a class of biological HIV infection model of latently infected cells using heuristic approach. Discret. Contin. Dyn. Syst. 2021, 14, 3611. [CrossRef]

2. Umar, M.; Sabir, Z.; Amin, F.; Guirao, J.L.G.; Raja, M.A.Z. Stochastic numerical technique for solving HIV infection model of CD4+ T cells. Eur. Phys. J. Plus 2020, 135, 405. [CrossRef]

3. Berge, T.; Lubuma, J.-S.; Moremedi, G.; Morris, N.; Kondera-Shava, R. A simple mathematical model for Ebola in Africa. J. Biol. Dyn. 2017, 11, 42-74. [CrossRef] [PubMed]

4. $\quad$ Bhola, J.; Venkateswaran, V.R.; Koul, M. Corona epidemic in Indian context: Predictive mathematical modelling. MedRxiv 2020. [CrossRef]

5. $\quad$ Li, Q.; Guan, X.; Wu, P.; Wang, X.; Zhou, L.; Tong, Y.; Ren, R.; Leung, K.S.; Lau, E.H.; Wong, J.Y.; et al. Early transmission dynamics in Wuhan, China, of novel coronavirus-infected pneumonia. N. Engl. J. Med. 2020, 382, 1199-1207. [CrossRef] [PubMed]

6. Bernasconi, A.; Grandi, S. A Conceptual Model for Geo-Online Exploratory Data Visualization: The Case of the COVID-19 Pandemic. Information 2021, 12, 69. [CrossRef]

7. Kucharski, A.J.; Russell, T.W.; Diamond, C.; Liu, Y.; Edmunds, J.; Funk, S.; Eggo, R.M.; Sun, F.; Jit, M.; Munday, J.D.; et al. Early dynamics of transmission and control of COVID-19: A mathematical modelling study. Lancet Infect. Dis. 2020, 20, 553-558. [CrossRef]

8. Spiteri, G.; Fielding, J.; Diercke, M.; Campese, C.; Enouf, V.; Gaymard, A.; Bella, A.; Sognamiglio, P.; Moros, M.J.S.; Riutort, A.N.; et al. First cases of coronavirus disease 2019 (COVID-19) in the WHO European Region, 24 January to 21 February 2020. Eurosurveillance 2020, 25, 2000178. [CrossRef] [PubMed]

9. Rhodes, T.; Lancaster, K. Mathematical models as public troubles in COVID-19 infection control: Following the numbers. Health Sociol. Rev. 2020, 29, 177-194. [CrossRef] [PubMed]

10. Benvenuto, D.; Giovanetti, M.; Vassallo, L.; Angeletti, S.; Ciccozzi, M. Application of the ARIMA model on the COVID-2019 epidemic dataset. Data Brief. 2020, 29, 105340. [CrossRef] [PubMed]

11. Mustafa, S.K.; Ahmad, M.A.; Sotnik, S.; Zeleniy, O.; Lyashenko, V.; Alzahrani, O. Brief review of the mathematical models for analyzing and forecasting transmission of COVID-19. J. Crit. Rev. 2020, 7, 4206-4210.

12. Sivakumar, A. Review of mathematical models to predict the rate of spread and control of COVID-19 in India. Bullet. World Health Organ. 2020. [CrossRef]

13. Nesteruk, I. Estimates of the COVID-19 pandemic dynamics in Ukraine based on two data sets. Medrxiv 2021. [CrossRef]

14. Thompson, R.N. Epidemiological models are important tools for guiding COVID-19 interventions. BMC Med. 2020, 18, 152. [CrossRef] [PubMed]

15. Libotte, G.B.; Lobato, F.S.; Platt, G.M.; Neto, A.J.S. Determination of an optimal control strategy for vaccine administration in COVID-19 pandemic treatment. Comput. Methods Programs Biomed. 2020, 196, 105664. [CrossRef] [PubMed]

16. Sadiq, I.Z.; Abubakar, F.S.; Dan-Iya, B.I. Role of nanoparticles in tackling COVID-19 pandemic: A bio-nanomedical approach. J. Taibah Univ. Sci. 2021, 15, 198-207. [CrossRef]

17. Gumel, A.B.; Iboi, E.A.; Ngonghala, C.N.; Elbasha, E.H. A primer on using mathematics to understand COVID-19 dynamics: Modeling, analysis and simulations. Infect. Dis. Model. 2021, 6, 148-168. [CrossRef] [PubMed] 
18. Ortenzi, F.; Albanese, E.; Fadda, M. A Transdisciplinary Analysis of COVID-19 in Italy: The Most Affected Country in Europe. Int. J. Environ. Res. Public Health 2020, 17, 9488. [CrossRef]

19. Sánchez, Y.G.; Sabir, Z.; Guirao, J.L.G. Design of a nonlinear sitr fractal model based on the dynamics of a novel coronavirus (COVID-19). Fractals 2020, 28, 2040026. [CrossRef]

20. Sabir, Z.; Umar, M.; Raja, M.A.Z.; Baleanu, D. Applications of gudermannian neural network for solving the sitr fractal system. Fractals 2021, 29. [CrossRef]

21. Moore, S.; Hill, E.M.; Tildesley, M.J.; Dyson, L.; Keeling, M.J. Vaccination and non-pharmaceutical interventions for COVID-19: A mathematical modelling study. Lancet Infect. Dis. 2021, 21, 793-802. [CrossRef]

22. Umar, Y. Theoretical studies of the rotational and tautomeric states, electronic and spectroscopic properties of favipiravir and its structural analogues: A potential drug for the treatment of COVID-19. J. Taibah Univ. Sci. 2020, 14, 1613-1625. [CrossRef]

23. Anirudh, A. Mathematical modeling and the transmission dynamics in predicting the Covid-19-What next in combating the pandemic. Infect. Dis. Model. 2020, 5, 366-374. [CrossRef]

24. Chen, X.; Zhang, A.; Wang, H.; Gallaher, A.; Zhu, X. Compliance and containment in social distancing: Mathematical modeling of COVID-19 across townships. Int. J. Geogr. Inf. Sci. 2021, 35, 446-465. [CrossRef]

25. Zhang, Z.; Zeb, A.; Hussain, S.; Alzahrani, E. Dynamics of COVID-19 mathematical model with stochastic perturbation. Adv. Differ. Equ. 2020, 2020, 451. [CrossRef] [PubMed]

26. Soumia, M.; Hanane, Z.; Benaissa, M.; Younes, F.Z.; Chakib, A.; Mohammed, B.; Mohamed, B. Towards potential inhibitors of COVID-19 main protease Mpro by virtual screening and molecular docking study. J. Taibah Univ. Sci. 2020, 14, 1626-1636. [CrossRef]

27. Bhadauria, A.S.; Pathak, R.; Chaudhary, M. A SIQ mathematical model on COVID-19 investigating the lockdown effect. Infect. Dis. Model. 2021, 6, 244-257. [CrossRef] [PubMed]

28. Yuanlei, S.; Almohsen, B.; Sabershahraki, M.; Issakhov, A.; Raja, M.A.Z. Nanomaterial migration due to magnetic field through a porous region utilizing numerical modeling. Chem. Phys. Lett. 2021, 785, 139162. [CrossRef]

29. Ghanbari, B.; Djilali, S. Mathematical analysis of a fractional-order predator-prey model with prey social behavior and infection developed in predator population. Chaos Solitons Fractals 2020, 138, 109960. [CrossRef]

30. Ghanbari, B.; Djilali, S. Mathematical and numerical analysis of a three-species predator-prey model with herd behavior and time fractional-order derivative. Math. Methods Appl. Sci. 2020, 43, 1736-1752. [CrossRef]

31. Bolboacă, S.D.; Jäntschi, L. Sensitivity, specificity, and accuracy of predictive models on phenols toxicity. J. Comput. Sci. 2014, 5, 345-350. [CrossRef]

32. Jäntschi, L.; Bolboacă, S.D.; Sestraş, R.E. Meta-heuristics on quantitative structure-activity relationships: Study on polychlorinated biphenyls. J. Mol. Model. 2010, 16, 377-386. [CrossRef] [PubMed] 